\title{
Estudio del Efecto Combinado del Secado y Freido en las Propiedades de Tortillas Tostadas
}

\author{
Jesús Morales-Pérez y Jorge F. Vélez-Ruiz* \\ Departamento de Ingeniería Química, Alimentos y Ambiental, Universidad de las Américas, Puebla \\ Ex hacienda. Sta. Catarina Mártir, Cholula, 72820 Puebla-México (e-mail: jorgef.velez@udlap.mx) \\ * autor a quien se debe dirigir la correspondencia
}

Recibido May. 31, 2010; Aceptado Jul. 15, 2010; Versión Final recibida Ago. 12, 2010

\begin{abstract}
Resumen
Se estudió el efecto del secado con aire previo al freído, en la disminución de grasa y en otras propiedades de tortillas tostadas. En el secado, se manejaron tres temperaturas $\left(35,48\right.$ y $\left.68{ }^{\circ} \mathrm{C}\right)$ y tres velocidades de aire $(2,4$ y $6 \mathrm{~m} / \mathrm{s})$, obteniendo una humedad en la tortilla de 6 a $12 \%$. En el proceso de freído, se analizaron tres temperaturas de aceite $\left(140,160\right.$ y $\left.180{ }^{\circ} \mathrm{C}\right)$ y cuatro tiempos de proceso $(30,60,90$ y $120 \mathrm{~s})$, partiendo de tortilla con $10 \%$ de humedad. Se determinó que una pérdida acelerada de humedad durante el secado resultó en una pérdida lenta de humedad en el freído y viceversa, y que el secado tuvo un efecto significativo sobre el freído. Las tostadas tuvieron un contenido de grasa más bajo que los productos comerciales, y atributos sensoriales semejantes. Se realizó una optimización del proceso mediante un programa computacional, basado en el modelo Box-Behnken y los resultados se compararon con datos experimentales.
\end{abstract}

Palabras clave: tortillas tostadas, secado, freído, combinación de procesos

\section{Study of the Combined Effect of Drying and Frying on Toasted Tortillas Properties}

\begin{abstract}
A study of the combination of air drying and immersion frying, and their effect on decreasing fat content and other properties of toasted tortillas was done. Drying with hot air $\left(35,48\right.$ and $\left.68^{\circ} \mathrm{C}\right)$ at 2,4 and $6 \mathrm{~m} / \mathrm{s}$ was utilized to dry the tortillas, in which the final moisture content was in a range of $6-12 \%$. Frying was conducted with initial moisture of $10 \%$ in the tortillas, at 140,160 and $180{ }^{\circ} \mathrm{C}$ and process times of $30,60,90$ and $120 \mathrm{~s}$ to get the toasted tortillas. Faster moisture loss during drying determined lower evaporation rates through the frying and vice versa. There was a significant effect of the drying conditions on the fried product. The toasted product had fat content lower than commercial products and similar quality attributes. Optimization of the process was carried out using a computer program and the Box-Behnken approach. Model results were compared with experimental data.
\end{abstract}

Keywords: toasted tortillas, drying, frying, process combination 


\section{INTRODUCCIÓN}

La demanda por los productos fritos sigue aumentando en México y Estados Unidos, con un crecimiento anual continuo en el volumen de ventas, su producción ha aumentado continuamente en México durante los años 2005-2008 (INEGI, 2009). Las botanas de maíz fritas son un producto muy popular en México y Estados Unidos, entre otros países; con un record de ventas únicamente superado por las papas fritas (Kawas y Moreira, 2001). Según la revista Alto Nivel (1999) se estima que en nuestro país la industria generadora de productos fritos tiene un valor de ventas de más de mil millones de pesos anuales, además posee uno de los índices más altos del mundo en el consumo de frituras.

Aunque las grasas son un constituyente esencial en la dieta humana por su aporte energético, su ingesta excesiva provoca problemas cardiovasculares y de obesidad. Los problemas más comunes relacionados con el consumo excesivo de grasa son principalmente ataques y paros cardiacos (Mellema, 2003; Sosa-Morales y Vélez-Ruiz, 2009). Lo anterior ha motivado a la industria de alimentos a buscar alternativas de producción de botanas para obtener productos alimenticios con una menor cantidad de grasa, pero conservando las características propias de los alimentos fritos, lo que ha motivado al diseño de nuevos procesos y a la combinación de los ya existentes, con el objetivo de obtener alimentos fritos con un bajo contenido de grasa (Moreira et al., 1995a; Esturk et al., 2000; Mellema, 2003; Sosa-Morales y Vélez-Ruiz, 2009). Las alternativas que han sido reportadas con el propósito de reducir el contenido de grasa en alimentos fritos, se han centrado en cuatro aspectos: i) modificación de la formulación o de la estructura de los alimentos a freir, ii) aplicación de películas comestibles sobre los alimentos, previamente a la inmersión en aceite, iii) sustitución o modificación del aceite de freído, y iv) reconsideración y modificación de los procesos de freído (Mellema, 2003; Moyano y Pedreschi, 2006; Sosa-Morales y Vélez-Ruiz, 2009).

Entre los estudios realizados sobre alguno de los cuatro aspectos mencionados se pueden citar los siguientes estudios: recubrimiento de diversos alimentos (pollo, botanas y donas) sujetos al proceso de freído, con películas comestibles (Balasubramaniam et al., 1997; Mittal y Zhang, 2000; García-Joffre et al., 2007); sustitución de manteca por aceites vegetales (Sinram y Hartman, 1989); restructuración de papas a la francesa para controlar la adsorción de aceite (Pinthus et al., 1992); incorporación de aditivos, jarabe y sal para modificación de las formulaciones utilizadas en la elaboración de papas fritas (Demetriades et al., 1995; Esturk et al., 2000; Moyano et al., 2002). Aplicación de vacío durante el proceso de freído (Garayo y Moreira, 2002); calentamiento por microondas y energía radiante (Lloyd et al., 2004), combinación de escaldado y freído (Moyano y Pedreschi, 2006) en la elaboración de papas a la francesa. Combinación de secado y freído en papas (Moyano y Pedreschi, 2006) y tostadas (Moreira et al., 1999; Morales-Pérez et al., 2006); combinación de vapor sobrecalentado y freído en placas de pollo (Moreira et al., 1999; Cenkowski et al., 2008).

De acuerdo a Moreira et al. (1997), la ganancia de aceite en productos fritos es mayor cuando el contenido de humedad es alto al inicio del proceso, porque al freír un alimento que ha sido reducido previamente en su contenido de humedad, hace que la absorción de aceite sea menor. Por otro lado, Gamble y Rice (1987) probaron diferentes métodos de secado (microondas, aire caliente y congelado) con el objetivo de disminuir el contenido de humedad inicial de papas a freir, encontrando que la mejor alternativa en el decremento de grasa ganada, fue el secado con aire caliente.

En el presente trabajo se planteó como objetivo, estudiar el efecto de ambos procesos, secado con aire y freído por inmersión en las propiedades de las tostadas, buscando reducir el contenido de grasa y conservar los otros atributos de calidad de este producto alimenticio. 


\section{MATERIALES Y MÉTODOS}

\section{Tortilla.}

Debido a la dificultad de mantener constantes las características de las muestras, se decidió trabajar con tortillas comerciales de maíz, de la marca Milpa Real, adquiridas en la localidad.

\section{Secado}

Para el secado con aire caliente se utilizó un secador de charolas a escala piloto, construido en la Universidad de las Américas Puebla, el cual está integrado por un ventilador (modelo CGP 13255, marca Koblenz), por tres válvulas de mariposa, y por resistencias eléctricas que permiten modificar el flujo y elevar la temperatura del aire. La velocidad de aire a la entrada del secador se determinó por medio de un anemómetro (LCA 6000, Airflow Instrumentation, RU), el equipo fue operado a 2, 4 y $6 \mathrm{~m} / \mathrm{s}$, mientras que la temperatura se midió con un termómetro de mercurio, operando el equipo a 35,48 y $68{ }^{\circ} \mathrm{C}$. La toma de muestras durante el secado, se realizó por periodos de 20 minutos, permitiendo el enfriamiento de la muestra a temperatura ambiente para pesarla y, colocarla dentro de bolsas herméticas de plástico para su procesamiento y análisis posterior.

\section{Freído}

El proceso de freído por inmersión se realizó en una freidora comercial (Rival ${ }^{\mathrm{MR}}$, Corea) con capacidad de $4 \mathrm{~L}$ de aceite, con resistencias eléctricas. La medición de temperatura se llevó a cabo por medio de un termómetro de mercurio, operando el equipo a 140,160 y $180{ }^{\circ} \mathrm{C}$. La toma de muestras se realizó cada $30 \mathrm{~s}$, permitiendo el atemperamiento, eliminando el exceso de grasa por medio de papel, y colocando la muestra en una bolsa hermética de plástico para los análisis posteriores. Se empleó aceite de maíz.

\section{Medición de Propiedades}

Contenido de Humedad. El contenido de humedad en la tortilla procesada, se determinó por pérdida de peso después de secar en una estufa convencional a $105^{\circ} \mathrm{C}$ por 12 horas (AOAC, 2000).

Contenido de Grasa. La determinación de grasa, se llevó a cabo mediante la extracción con éter de petróleo (AACC, 1986), usando el sistema Goldfish (Labconco Corporation, Kansas City, Missouri).

Análisis de Textura. La propiedad usada para expresar la textura de las muestras, fue la crujencia, como un atributo que denota frescura y calidad (Bourne, 2002). Esta propiedad fue medida como la fuerza requerida para la ruptura de la muestra, por medio de un texturómetro (Texture Analyzer $^{\mathrm{TM}}$, modelo TA-XT2, Texture Technologies Corporation, Scardale, NY). La prueba involucró la aplicación de una fuerza directa sobre la muestra, mediante un embolo esférico de $1,25 \mathrm{~cm}$. de diámetro a una velocidad de descenso de $2,0 \mathrm{~mm} / \mathrm{s}$. La tostada permaneció colocada sobre una base (cilindro hueco de 5,5 y $6,0 \mathrm{~cm}$ de diámetros interno y externo, respectivamente), la muestra se fracturó y se registró el valor máximo o pico que representa la fuerza. La textura en las tostadas fue medida en muestras recién hechas, permitiendo su atemperamiento y/o su eliminación de exceso de aceite.

Análisis de Color. El color se determinó utilizando un colorímetro Color Gard System en modo de reflectancia (Gardner Colorgard System/05, Reston, VA), midiendo los parámetros $L$, a y b de la escala de Hunter, previamente calibrado con los mosaicos negro y blanco $(L=92,89, a=1,05$ y $b=0,82)$. El cambio neto de color se determinó mediante la relación:

$$
\Delta E=\sqrt{\left(L_{t}-L_{r}\right)^{2}+\left(a_{t}-a_{r}\right)^{2}\left(b_{t}-b_{r}\right)^{2}}
$$

Donde: $L$ es la luminosidad, $b$ es el índice del color amarillo-azul, y a es el parámetro del color rojo-verde, para cualquier tiempo o condición de proceso $(t)$ y al inicio del proceso o valor de referencia $(r)$. 
Las pruebas mencionadas se realizaron por triplicado en la materia prima y en las tostadas comerciales, y solo se hicieron por duplicado en las muestras tomadas durante el secado y el freído.

\section{Análisis Sensorial}

Se analizaron color, sabor, textura y aceptación general de las tostadas, mediante la aplicación de una escala hedónica y de una comparación duo-trio (Lardmon, 1970). Con un grupo de 20 panelistas no entrenados, a quienes se les proporcionó una ficha de evaluación, con una escala de nueve puntos, correspondiendo 1 a me disgusta extremadamente, y 9 a me gusta extremadamente.

\section{Análisis de Datos y Optimización}

El análisis estadístico de datos se realizó empleando un paquete Minitab 2000 (versión 13,20, Minitab Inc., State College, PA), mediante una prueba de ANOVA con un nivel de confianza del $95 \%$, y en la comparación por pares se empleo la prueba de Tukey. Mientras que para la optimización y determinación de los polinomios de respuesta, se utilizó un paquete estadístico Desing Expert 2002 (versión 6,0,6, Stat-Ease Inc. Minneapolis, MN) empleando el modelo de BoxBehnken.

\section{RESULTADOS Y DISCUSIÓN}

\section{Caracterízación de Tortillas y Tostadas Comerciales}

Las propiedades de las tortillas como materia prima (marca Milpa Real) que fueron determinadas en el laboratorio, se muestran en la Tabla 1.

Tabla 1. Características de la tortilla (materia prima).

\begin{tabular}{|c|c|c|c|c|c|c|}
\hline \multicolumn{2}{|c|}{ Componente/ Propiedad } & Valor & \multicolumn{3}{|c|}{ Componente/ Propiedad } & Valor \\
\hline Humedad & \multirow{2}{*}{\multicolumn{2}{|c|}{ 1,99 $\pm 0,12$ B.H. (\%) }} & \multicolumn{2}{|c|}{ Fuerza de ruptura } & \multicolumn{2}{|c|}{$40,50 \pm 0,71(\mathrm{~N})$} \\
\hline Grasa & & & Color: & $\mathrm{L}$ & \multicolumn{2}{|c|}{$72,85 \pm 1,29$} \\
\hline Espesor & \multicolumn{2}{|c|}{$1,93 \pm 0,08(\mathrm{~m})$} & & a & \multicolumn{2}{|c|}{$-1,85 \pm 0,04$} \\
\hline Diámetro & \multicolumn{2}{|c|}{$14,50 \pm 0,00(\mathrm{~m})$} & & $b$ & 13 & $7 \pm 0,06$ \\
\hline
\end{tabular}

La composición de esta tortilla (humedad y grasa) está por arriba de lo reportado para una tortilla promedio de maíz (44.8\% y $1.5 \%$, respectivamente) por el Instituto Nacional de la Nutrición de México (Hernández et al., 1974); sin embargo estos contenidos como el resto de las determinaciones físicas, se pueden considerar que son propias y representativas de este alimento. Por otro lado y para tener un marco de referencia, se realizó una comparación de algunas propiedades para tostadas correspondientes a cuatro marcas comerciales mexicanas (Charras, Delicias, Milpa Real y Totopos), encontrando que los productos seleccionados contienen entre 1,8 y $6,7 \%$ de humedad, que son semejantes a los reportados $(1,2-3,0 \%)$ por Moreira et al. (1995a, 1995b, 1995c). Por medio del análisis de varianza se determinó que las cuatro muestras comerciales analizadas presentaron diferencia significativa $(p<0,05)$ entre sí, en lo que a humedad se refiere.

La determinación del contenido de grasa en las tostadas comerciales resultó en un rango amplio de 24 a 42\%; lo que contribuyó a encontrar una diferencia significativa $(p<0,05)$ entre marcas. El contenido de grasa (22 a 28\%) en tres marcas analizadas (Charras, Delicias, y Milpa Real) es similar a los chips de tortilla reportados por Moreira et al. (1995a, 1995b, 1995c) de 20 a 28\%. Por lo que se puede decir que los materiales caracterizados, con excepción de la marca Totopos (grasa $>35 \%$ ), contienen una cantidad de grasa propia de este tipo de productos fritos.

En cuanto al color de las muestras, la luminosidad ( $L: 23-25)$ y el grado de amarillez (b: 1,3-3,3) si resultaron diferentes al $95 \%$ de confianza, mientras que el parámetro rojizo (a: $0,04-0,18)$ no mostró diferencia significativa $(p>0,05)$ entre marcas comerciales. Por otro lado, la fuerza de 
ruptura osciló entre 5,8-7,6 N; que es semejante al valor reportado Moreira et al. (1995a), con fuerzas de ruptura de 6,0 N para totopos; esta propiedad no mostró diferencia significativa entre marcas, y solo se observó que a un mayor porcentaje de humedad le correspondió una mayor fuerza de ruptura.

\section{Secado de Tortillas}

Debido a la variación en el contenido de humedad de las tortillas, como resultado del empleo de materia prima proveniente de diferentes lotes, y para no incluirla como una variable de estudio, se decidió controlar la humedad inicial de la tortilla antes del proceso de secado. Dicha humedad fue de $40 \%$, valor que correspondió a la humedad mínima determinada experimentalmente. El ajuste de humedad se realizó con el mismo equipo de secado empleando como condiciones de operación $2 \mathrm{~m} / \mathrm{s}$ y $48^{\circ} \mathrm{C}$, que fueron condiciones seleccionadas en base a experimentos previos.

Después de realizar el ajuste de humedad inicial en la tortilla, se realizaron las corridas de secado a tres velocidades $(2,4$ y $6 \mathrm{~m} / \mathrm{s})$ y a tres temperaturas $\left(35,48\right.$ y $\left.68^{\circ} \mathrm{C}\right)$ del aire, monitoreando el contenido de humedad en la tortilla con respecto al tiempo, para obtener las curvas de secado. El contenido de humedad en la materia prima se redujo desde 40 hasta 10\% (b.h.) con el propósito de determinar el efecto de la velocidad y la temperatura del aire, para una pérdida de humedad semejante. La Figura 1 incluye las tres curvas de secado para la deshidratación con aire a $48{ }^{\circ} \mathrm{C}$, de manera semejante se obtuvieron las curvas correspondientes a las otras dos temperaturas.

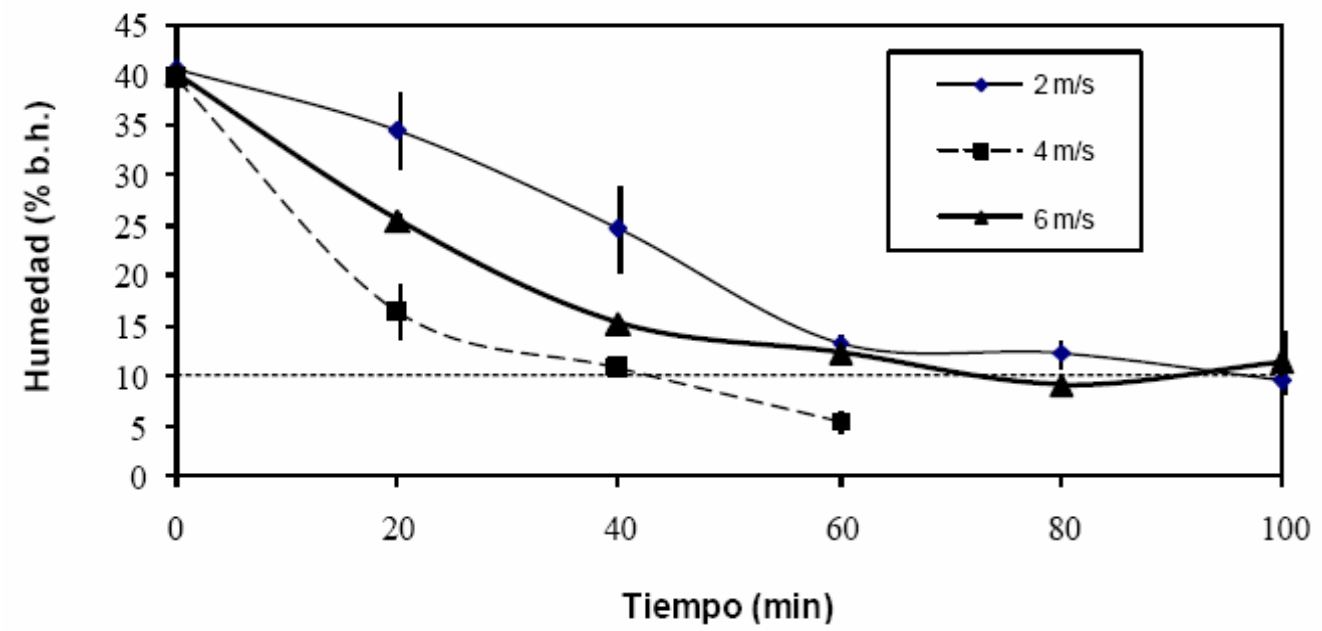

Fig. 1: Curvas de secado para tortilla a $48^{\circ} \mathrm{C}$

En general, la rapidez de pérdida de humedad incrementó al aumentar la velocidad y la temperatura del aire, pero inesperadamente a $48{ }^{\circ} \mathrm{C}$ resultó una humedad menor para la corriente de aire intermedia $(4 \mathrm{~m} / \mathrm{s})$. A partir de las curvas de secado correspondientes a las diferentes condiciones de operación experimentadas, se determinaron los tiempos de proceso para alcanzar el $10 \%$ de humedad de la tortilla en el secador. Para las curvas mostradas a $48{ }^{\circ} \mathrm{C}$ (Fig. 1), resultaron en una magnitud de 95, 42 y 71 minutos para las velocidades de 2,4 y $6 \mathrm{~m} / \mathrm{s}$, respectivamente.

Como se puede apreciar, la velocidad de $4 \mathrm{~m} / \mathrm{s}$ implicó un menor tiempo de proceso; lo cual no se observó a las otras temperaturas. Esta situación de menor pérdida de humedad para una velocidad de aire mayor, se puede relacionar a la formación de una costra superficial; al secar la tortilla demasiado rápido con aire a $6 \mathrm{~m} / \mathrm{s}$; por lo que se limitó la salida de agua de la muestra por el efecto de la resistencia superficial adicional de dicha barrera, disminuyendo así la velocidad de secado, aún cuando el contenido de agua sea alto (Treybal, 1980; Okos et al., 1992; Rahman y Perera, 2007). Los tiempos de secado determinados experimentalmente y correspondientes a las diferentes condiciones de operación estudiadas, para lograr una humedad de $10 \%$ en la tortilla, se incluyen en la Tabla 2. Esta anomalía observada en los tiempos de secado, solo resultó a $48{ }^{\circ} \mathrm{C}$. 
Tabla 2. Tiempos de secado correspondientes a las diferentes condiciones de operación, para un contenido de $10 \%$ de humedad en la tortilla.

\begin{tabular}{clll}
\hline Velocidad del aire & $35^{\circ} \mathrm{C}$ & $48^{\circ} \mathrm{C}$ & $68^{\circ} \mathrm{C}$ \\
\hline $2 \mathrm{~m} / \mathrm{s}$ & $200 \mathrm{~min}$ & $95 \min$ & $55 \mathrm{~min}$ \\
$4 \mathrm{~m} / \mathrm{s}$ & 174 & 42 & 45 \\
$6 \mathrm{~m} / \mathrm{s}$ & 172 & 71 & 30 \\
\hline
\end{tabular}

En esta tabla, se puede apreciar que los tiempos necesarios para disminuir la humedad al $10 \%$, resultaron semejantes para 4 y $6 \mathrm{~m} / \mathrm{s}$ a $35^{\circ} \mathrm{C}$, correspondientes a la velocidad en la temperatura alta (menor tiempo a mayor velocidad), e inversamente proporcionales a la velocidad a $48{ }^{\circ} \mathrm{C}$ (menor tiempo a menor velocidad) En lo que respecta a la fuerza de rompimiento de las tortillas en diferentes momentos de la deshidratación con aire, se observó que al inicio la tortilla es blanda y prácticamente no ofrece resistencia $(<3 \mathrm{~N})$. La fuerza se incrementó, hasta alcanzar su mayor resistencia alrededor de los 60 minutos de secado (15 a $21 \mathrm{~N}$ ), mostrando una mayor resistencia con una mayor velocidad de secado; posteriormente la fuerza disminuyó hasta resultar indiferente al efecto de la velocidad del aire; al final de un proceso largo (120 min) se registró una resistencia prácticamente constante de $5 \mathrm{~N}$. En general se observó que el manejo de velocidades bajas (2 y 4 $\mathrm{m} / \mathrm{s}$ ) contribuyó a generar un producto más blando, lo que se puede considerar como un efecto positivo. La fuerza de ruptura obtenida al secar las tortillas a las diferentes temperaturas (Figura 2), incluyen las muestras obtenidas a las tres velocidades, mostrando un rango de 8 a $12 \mathrm{~N}$ como valor promedio.

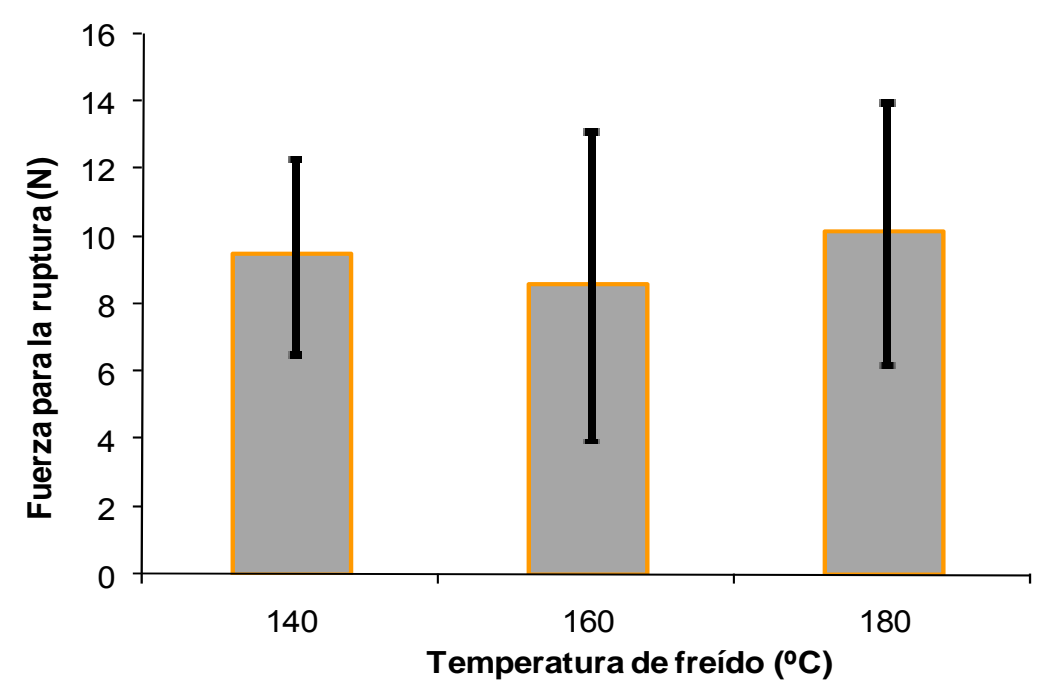

Fig. 2: Fuerza de ruptura de tortilla secada con aire a tres temperaturas.

\section{Freído de Tortillas Secas}

\section{Efecto del Secado (Velocidad del Aire)}

Las tortillas secas con alrededor de $10 \%$ de humedad fueron sometidas al proceso de freído por inmersión en aceite, mostrando una evolución de parámetros variable con respecto a la temperatura de freído. La tabla 3 resume la cinética de la humedad y grasa durante el proceso de freído para las tortillas deshidratadas a $48{ }^{\circ} \mathrm{C}$, resultados semejantes fueron obtenidos para las otras dos temperaturas de secado. La humedad final, después de $120 \mathrm{~s}$ de freído, varió entre 1,7 y $7,3 \%$ (b.h.) dependiendo de las condiciones del proceso de freído. Mientras que la grasa tuvo un rango de 9.1 a $17.3 \%$, dependiendo de las condiciones de operación.

La velocidad de aire empleada en el secado si generó diferencia significativa $(p<0,05)$ en el contenido de humedad final del producto frito, dicha diferencia existió para cada tiempo en el que se realizó el muestreo $(30,60,90$ y 120 s); en dicha tabla se aprecia una disminución mayor de 
humedad, cuando la tortilla fue secada con bajas velocidades de aire, excepto a $180^{\circ} \mathrm{C}$. Mediante la prueba de Tukey, se encontró que la diferencia solo existió entre 2 y $6 \mathrm{~m} / \mathrm{s}$, al igual que entre 4 y $6 \mathrm{~m} / \mathrm{s}$, lo que refuerza la idea de un posible encostramiento en la tortilla, desarrollado durante el secado a la velocidad alta y que posteriormente influyó en la pérdida de humedad durante el freído.

De manera análoga que en la humedad, la velocidad de secado afectó significativamente $(p<$ $0,05)$ el contenido de grasa del producto frito. De la prueba de comparación por pares, se determinó que solo existió diferencia cuando se empleó la velocidad de $4 \mathrm{~m} / \mathrm{s}$, es decir que entre 2 y $6 \mathrm{~m} / \mathrm{s}$ no hubo diferencia significativa. Los valores más altos en grasa para la tostada, correspondieron al secado a $4 \mathrm{~m} / \mathrm{s}$. Estos resultados concuerdan con lo reportado por Moreira et al. (1998), que mencionan que el contenido de aceite para totopos fue afectado por el contenido de humedad inicial, un alto contenido inicial de humedad generó una alta ganancia de aceite, además de la influencia de otros factores.

En cuanto al cambio neto de color $(\Delta \mathrm{E})$, se calculó un rango final de 10 a 12 (Figura 3), lo cual arrojó una diferencia significativa $(p<0,05)$ entre muestras, debido a la velocidad de aire en el secador; este parámetro mostró una relación inversa con respecto a la velocidad de aire, es decir el mayor $\Delta \mathrm{E}$, le correspondió al secado a $2 \mathrm{~m} / \mathrm{s}$ y viceversa. Al comparar los parámetros del color de manera individual, se determinó un efecto significativo $(p<0,05)$ de la velocidad del aire sobre los parámetros $b$ y $a$, pero no sobre $L$, lo cual tiene relación con cierto grado de oscurecimiento desarrollado durante el freído, que influye en los componentes rojo y amarillo, y poco en luminosidad.

Tabla 3. Evolución de la humedad $(H)$ y grasa $(G)$ de la tortilla (\% b.h.) durante el proceso de freído.

\begin{tabular}{|c|c|c|c|c|c|c|c|}
\hline $\mathrm{T}\left({ }^{\circ} \mathrm{C}\right)$ & & & 0 & $30 \mathrm{~s}$ & $60 \mathrm{~s}$ & $90 \mathrm{~s}$ & $120 \mathrm{~s}$ \\
\hline \multirow[t]{6}{*}{140} & secada a $2 \mathrm{~m} / \mathrm{s}$ & $\mathrm{H}:$ & $10,72 \pm 0,73$ & $6,41 \pm 0,08$ & $5,77 \pm 0,15$ & $4,88+0,30$ & $4,23+0,07$ \\
\hline & & G: & $0,62+0,01$ & $10,02 \pm 0,16$ & $10,00+0,01$ & $9,54+1,13$ & $9,73+0,07$ \\
\hline & secada a $4 \mathrm{~m} / \mathrm{s}$ & $\mathrm{H}:$ & $10,67 \pm 0,05$ & $6,52 \pm 0,57$ & $5,60+0,26$ & $4,88+0,24$ & $4,61+0,19$ \\
\hline & & G: & $0,64+0,01$ & $10,11+0,16$ & $14,91+0,59$ & $16,86+0,51$ & $15,41+2,72$ \\
\hline & secada a $6 \mathrm{~m} / \mathrm{s}$ & $\mathrm{H}$ : & $10,34 \pm 0,15$ & $7,91+0,28$ & $7,62 \pm 0,00$ & $7,39+0,22$ & $7,30 \pm 0,29$ \\
\hline & & G: & $0,63+0,01$ & $8,34+0,86$ & $8,41+0,40$ & $8,31+0,41$ & $9,14+0,67$ \\
\hline \multirow[t]{6}{*}{160} & secada a $2 \mathrm{~m} / \mathrm{s}$ & $\mathrm{H}$ : & $10,72+0,73$ & $4,89+0,02$ & $3,24+0,04$ & $1,97 \pm 0,04$ & $2,29+0,08$ \\
\hline & & G: & $0,62+0,01$ & $9,02+1,20$ & $9,55 \pm 1,45$ & $10,57+0,17$ & $10,66+0,09$ \\
\hline & secada a $4 \mathrm{~m} / \mathrm{s}$ & $\mathrm{H}:$ & $10,72 \pm 0,73$ & $4,62 \pm 0,03$ & $3,96 \pm 0,02$ & $2,83+0,07$ & $2,29+0,08$ \\
\hline & & G: & $0,64+0,01$ & $12,69+0,28$ & $13,95 \pm 0,83$ & $16,64+0,13$ & $16,99+0,04$ \\
\hline & secada a $6 \mathrm{~m} / \mathrm{s}$ & $\mathrm{H}:$ & $10,34+0,15$ & $5,84+0,09$ & $4,24+0,33$ & $3,91+0,51$ & $3,84+0,51$ \\
\hline & & G: & $0,63 \pm 0,01$ & $11,70+3,38$ & $7,12 \pm 0,03$ & $7,42+0,67$ & $9,80 \pm 1,31$ \\
\hline \multirow[t]{6}{*}{180} & secada a $2 \mathrm{~m} / \mathrm{s}$ & $\mathrm{H}$ : & $10,72+0,73$ & $3,94+0,11$ & $3,13+0,37$ & $2,83+0,75$ & $2,45+0,24$ \\
\hline & & G: & $0,62 \pm 0,01$ & $8,51+0,28$ & $9,24 \pm 0,74$ & $10,57 \pm 0,11$ & $10,71+0,08$ \\
\hline & secada a $4 \mathrm{~m} / \mathrm{s}$ & $\mathrm{H}:$ & $10,67 \pm 0,73$ & $3,94+0,11$ & $3,13+0,37$ & $2,83+0,75$ & $2,45 \pm 0,24$ \\
\hline & & G: & $0,64+0,01$ & $12,02+0,65$ & $12,54+0,25$ & $15,37 \pm 1,77$ & $17,27+0,41$ \\
\hline & secada a $6 \mathrm{~m} / \mathrm{s}$ & $\mathrm{H}:$ & $10,34+0,15$ & $5,27 \pm 1,07$ & $3,07+0,59$ & $2,34+0,39$ & $1,74+0,25$ \\
\hline & & G: & $0,63 \pm 0,01$ & $7,74+0,28$ & $10,31+0,01$ & $10,09+1,26$ & $10,94+0,38$ \\
\hline
\end{tabular}

Finalmente, la textura presentó una influencia significativa $(p<0,05)$ de la velocidad de aire de secado, el producto frito y secado previamente a $4 \mathrm{~m} / \mathrm{s}$ registró una media (a las tres temperaturas) de $5,51 \pm 0,45 \mathrm{~N}$ que correspondió a un producto mas fracturable en comparación con las otras dos velocidades empleadas a las mismas temperaturas $(11,64 \pm 2,30 \mathrm{~N}$ para $2 \mathrm{~m} / \mathrm{s}$, y $6,74 \pm 0,72 \mathrm{~N}$ para $6 \mathrm{~m} / \mathrm{s}$ ). Al considerar la fuerza de ruptura para las tostadas de 4 y $6 \mathrm{~m} / \mathrm{s}$, son las que se aproximan más a la fuerza registrada para los productos comerciales $(5,79$ a 7,55 N). 
La tortilla de maíz frita mostró mayor humedad cuando se empleó $35^{\circ} \mathrm{C}$ en el secado. De manera similar, la temperatura del aire afectó el contenido de grasa en el producto terminado, cuando se manejaron temperaturas altas del aire, se obtuvo un producto con mayor cantidad de grasa. También se observó que la fuerza de ruptura de las tostadas $(11.5 \pm 3.3$ vs. $10.9 \pm 3.3 \mathrm{~N})$ que fueron secadas a 35 y $48{ }^{\circ} \mathrm{C}$ resultó estadísticamente igual; de la misma manera que cuando se comparó la fuerza $(10.9 \pm 3.3$ vs. $9.6 \pm 3.5 \mathrm{~N})$ de tostadas secadas a 48 y $68{ }^{\circ} \mathrm{C}$. Moreira et al. (1995a), reportan la un rango de fracturabilidad de 2 a $14 \mathrm{~N}$, como función del tiempo de freído a $190{ }^{\circ} \mathrm{C}$. La temperatura del aire afectó significativamente $(p<0.05)$ tanto la humedad, la grasa, como la resistencia de las tostadas, pero no tuvo un efecto importante sobre el color, en ninguno de sus parámetros.

\section{Efecto de la Temperatura y del Tiempo de Freído}

Aunque se reconoce la influencia de los tratamientos previos, se puede considerar a la temperatura del aceite de freído como la variable mas importante y determinante en las características finales de las tostadas. Se puede observar (Tabla 3) que la diferencia entre el uso de temperaturas de 160 y $180^{\circ} \mathrm{C}$ no generó un cambio notable en el contenido de humedad $(2.3$ y $2.5 \%$ para $2 \mathrm{~m} / \mathrm{s}$ y $4 \mathrm{~m} / \mathrm{s}, 3.8$ y 1.7 para $6 \mathrm{~m} / \mathrm{s}$, respectivamente), mientras que la humedad final resultó mayor a $140^{\circ} \mathrm{C}$. Las velocidades de evaporación fueron más altas a 160 y $180{ }^{\circ} \mathrm{C}$. Como se esperaba, el contenido de humedad final en el producto mostró una influencia significativa de dicha temperatura $(p<0.05)$, al comparar 140 y $180^{\circ} \mathrm{C}$, y una situación semejante sucede entre 140 y $160^{\circ} \mathrm{C}$.

En lo que respecta a las otras características, se encontró que la temperatura del aceite si afectó significativamente el cambio neto de color, así como en los parámetros de color rojizo (a) y amarillento (b). Por otro lado, no existió diferencia significativa $(p>0.05)$ en el contenido de grasa por efecto de la temperatura de freído; observación que concuerda con lo reportado por Moreira et al. (1997) quienes determinaron que el contenido final de aceite en tortillas chips fue independiente de la temperatura de freído. En la textura tampoco hubo efecto significativo de la temperatura de freído, y en general, la fuerza de ruptura resultó mayor cuando el alimento fue freído a una temperatura de aceite mas alta $(9.5 \pm 2.9,8.6 \pm 4.5$ y $10.2 \pm 3.9 \mathrm{~N}$ a 140,160 y $180{ }^{\circ} \mathrm{C}$, respectivamente). Este resultado concuerda con lo reportado por Garayo y Moreira (2002) quienes afirman que la fuerza requerida para romper una tortilla chip incrementó al aumentar la temperatura de freído, aunque ellos obtuvieron valores mas bajos de dureza $(<3.8 \mathrm{~N})$.

Con excepción del contenido de humedad que disminuyó al transcurrir el tiempo de freído, los otros parámetros medidos como la grasa, el cambio neto de color, los componentes del color a, b y la textura muestran una tendencia de aumento con respecto al tiempo de proceso, hasta que el alimento alcanza cierto equilibrio y adquiere las características propias de los alimentos fritos. En la Figura 4, se puede observar que después de $90 \mathrm{~s}$, la disminución de humedad es muy lenta, además el alimento comienza a tomar propiedades físicas (sabor, color y textura) que pueden llegar a ser inaceptables por el consumidor. El tiempo de proceso tuvo un efecto significativo ( $p<$ 0.05 ) en humedad, grasa, grado de rojizo y amarillez, cambio neto de color, y textura; y solo no influyó en la luminosidad.

\section{Transferencia de Masa}

Analizando algunos aspectos adicionales del transporte de masa desarrollado durante la combinación de ambos procesos, se tienen las siguientes observaciones.

\section{Secado a Velocidad Constante}

En términos generales la evaporación de agua durante la etapa de secado mostró un incremento al aumentar la temperatura y la velocidad del aire, excepto a $48{ }^{\circ} \mathrm{C}$. En todas las curvas de secado se registraron dos periodos de secado (Figura 1); el tiempo correspondiente al periodo de velocidad constante disminuyó al aumentar la temperatura y la velocidad de aire en el secador. 
Los tiempos correspondientes a este periodo, así como las razones de agua evaporada calculadas, son los siguientes: $80 \mathrm{~min}-0.42 \mathrm{~g} / \mathrm{min}(2 \mathrm{~m} / \mathrm{s}), 60 \mathrm{~min}-0.48 \mathrm{~g} / \mathrm{min}(4 \mathrm{~m} / \mathrm{s}), 40 \mathrm{~min}-0.63$ $\mathrm{g} / \mathrm{min}(6 \mathrm{~m} / \mathrm{s})$ a $35^{\circ} \mathrm{C} ; 60 \mathrm{~min}-0.45 \mathrm{~g} / \mathrm{min}(2 \mathrm{~m} / \mathrm{s}), 30 \mathrm{~min}-0.90 \mathrm{~g} / \mathrm{min}(4 \mathrm{~m} / \mathrm{s}), 40 \mathrm{~min}-0.63 \mathrm{~g} / \mathrm{min}(6$ $\mathrm{m} / \mathrm{s})$ a $48{ }^{\circ} \mathrm{C} ; 40 \mathrm{~min}-1.06 \mathrm{~g} / \mathrm{min}(2 \mathrm{~m} / \mathrm{s}), 20 \mathrm{~min}-1.11 \mathrm{~g} / \mathrm{min}(4 \mathrm{~m} / \mathrm{s}), 20 \mathrm{~min}-1.23 \mathrm{~g} / \mathrm{min}(6 \mathrm{~m} / \mathrm{s})$ a $68^{\circ} \mathrm{C}$.

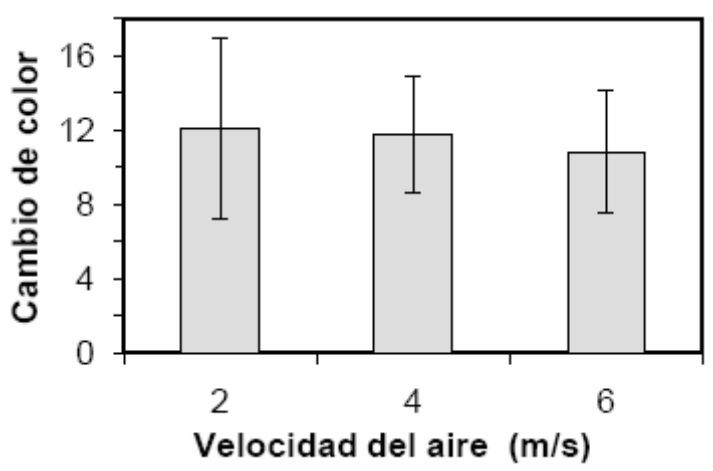

Fig. 3: Efecto de la velocidad del aire en el cambio neto de color de las tostadas.

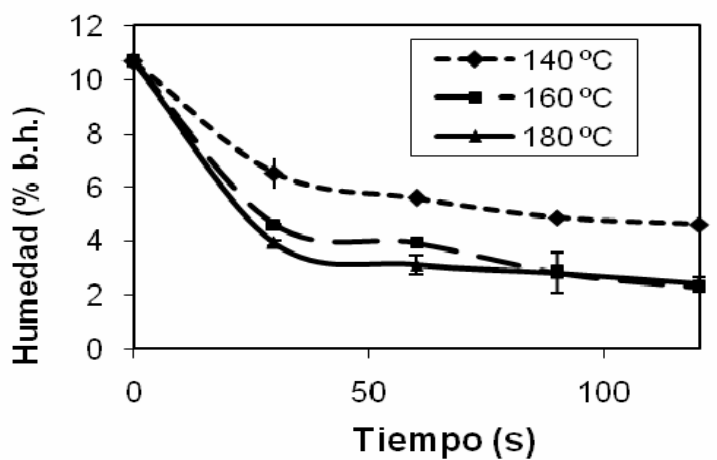

Fig. 4: Cambio de humedad durante el freído de tortilla a las tres temperaturas (secadas a 4 $\mathrm{m} / \mathrm{s})$.

\section{Proceso de Freído}

Para la etapa de freído, se observó que en los primeros 30 segundos al utilizar temperaturas de aceite de 160 y $180{ }^{\circ} \mathrm{C}$ la pérdida de agua fue mayor; fenómeno que no es tan claro para la ganancia de grasa, como se puede notar en la Tabla 3. En este periodo de evaporación constante se calculó un flujo medio de $0.20 \mathrm{~g}$ de agua por segundo. A $140{ }^{\circ} \mathrm{C}$ se notó un comportamiento diferente con menor eliminación de agua. Como se mencionó anteriormente, el secado influyó en la rapidez con la que el agua fue retirada del alimento durante este proceso, con velocidades diferentes.

El freído posterior a $30 \mathrm{~s}$, se consideró como un periodo de deshidratación decreciente, donde el coeficiente de difusión fue calculado en base a la segunda ley de Fick (un solo término de la serie) y considerando un espesor en la tortilla de $0.923 \mathrm{~mm}$ (en el secado el espesor considerado fue de $0.965 \mathrm{~mm}$ ), la mayoría de los valores obtenidos se muestran en la Tabla 4. Este coeficiente aumentó al incrementar la temperatura de freído y disminuyó con el incremento en la velocidad de aire; pero no es un patrón bien definido y, las variaciones son mas notables en muestras secadas a $48{ }^{\circ} \mathrm{C}$.

Tabla 4: Coeficientes de humedad $(D)^{*}$ durante el freído de tortilla, previamente secada.

\begin{tabular}{llllllllllll}
\hline Secado $\mathrm{T}\left({ }^{\circ} \mathrm{C}\right)$ & 35 & 35 & 35 & 48 & 48 & 48 & 68 & 68 & 68 & 35 & 35 \\
Velocidad $(\mathrm{m} / \mathrm{s})$ & 2 & 4 & 6 & 2 & 4 & 6 & 2 & 4 & 6 & 2 & 4 \\
T de freído $\left({ }^{\circ} \mathrm{C}\right)$ & 140 & 140 & 140 & 140 & 140 & 140 & 140 & 140 & 160 & 160 & 160 \\
$\mathrm{D} \times 10^{9}\left(\mathrm{~m}^{2} / \mathrm{s}\right)$ & 3,30 & 2,62 & 2,51 & 3,04 & 3,14 & 1,75 & 1,89 & 2,62 & 2,22 & 3,20 & 2,50 \\
& & & & & & & & & & & \\
Secado T $\left({ }^{\circ} \mathrm{C}\right)$ & 35 & 48 & 48 & 48 & 68 & 68 & 68 & 48 & 48 & 48 \\
Velocidad $(\mathrm{m} / \mathrm{s})$ & 6 & 2 & 4 & 6 & 2 & 4 & 6 & 2 & 4 & 6 \\
T de freído $\left({ }^{\circ} \mathrm{C}\right)$ & 160 & 160 & 160 & 160 & 160 & 160 & 160 & 180 & 180 & 180 \\
$\mathrm{D} \times 10^{9}\left(\mathrm{~m}^{2} / \mathrm{s}\right)$ & 2,35 & 3,91 & 4,40 & 3,84 & 3,23 & 2,50 & 3,59 & 3,39 & 3,14 & 4,40
\end{tabular}

ॠ Control: $\left(\mathrm{D}=3,20\right.$ a $160^{\circ} \mathrm{C} ; 13.8$ a $\left.180^{\circ} \mathrm{C}\right) \times 10^{-9}$

Los valores obtenidos de este coeficiente resultaron en un rango de 1.75 a $4.40 \times 10^{-9} \mathrm{~m}^{2} / \mathrm{s}$, que en general fue menor a $180^{\circ} \mathrm{C}$ en comparación con el control (tortilla sin secar), pero no sucedió lo mismo a $160{ }^{\circ} \mathrm{C}$; obviamente debido a la menor cantidad de agua presente en la tortilla de humedad controlada. Se observó que bajas velocidades de aire provocaron fluctuaciones en el coeficiente de difusión, contrario al secado a $6 \mathrm{~m} / \mathrm{s}$, en donde el coeficiente difusional promedio aumentó con el incremento en la temperatura del aceite $\left(2.16\right.$ a $140,3.26$ a 160 y $4.40 \times 10^{-9} \mathrm{~m}^{2} / \mathrm{s}$ 
a $\left.180^{\circ} \mathrm{C}\right)$. El valor difusional mas bajo $\left(1.75 \times 10^{-9} \mathrm{~m}^{2} / \mathrm{s}\right)$ correspondió a la muestra secada a $48^{\circ} \mathrm{C}$ $6 \mathrm{~m} / \mathrm{s}$ y freída a $140{ }^{\circ} \mathrm{C}$; mientras que un valor alto $\left(4.40 \times 10^{-9} \mathrm{~m}^{2} / \mathrm{s}\right)$ se obtuvo con las muestras secadas a dos condiciones: $48^{\circ} \mathrm{C}-4 \mathrm{~m} / \mathrm{s}$ y freída a $160{ }^{\circ} \mathrm{C}$ por un lado, y $48^{\circ} \mathrm{C}-6 \mathrm{~m} / \mathrm{s}$ y freída a $180^{\circ} \mathrm{C}$, por otro. Estos valores son similares a los reportados por Sosa et al. (2005) para el freído de carnitas de puerco (1.59 a $\left.30.20 \times 10^{-9} \mathrm{~m}^{2} / \mathrm{s}\right)$, Math et al. (2003) en el freído de papad (2.83 a $\left.5.03 \times 10^{-9} \mathrm{~m}^{2} / \mathrm{s}\right)$, Rubnov y Saguy (1997) para el freído de papas reestructuradas $(1.26$ a $2.00 \mathrm{x}$ $10^{-9} \mathrm{~m}^{2} / \mathrm{s}$ ), y menores a los reportados por Moreira et al. (1995b) para el freído de tortilla chips a $190{ }^{\circ} \mathrm{C}\left(9.35 \times 10^{-8} \mathrm{~m}^{2} / \mathrm{s}\right)$. Aunque los valores son comparables, las diferencias en magnitud se deben al tipo de alimento, humedad presente, aceite empleado, características estructurales y área de transferencia del alimento, así como a las condiciones de operación del proceso utilizadas en cada experimento.

\section{Optimización y Simulación de los Parámetros de Operación}

Al aplicar la metodología de superficies de respuesta al diseño experimental usado en nuestro estudio con los resultados correspondientes y mediante el modelo de Box Behnken, por medio del programa estadístico Desing Expert 6.0.6, se obtuvieron las ecuaciones de optimización para algunos de los parámetros medidos (en base a valores codificados $-1,0$ y 1 , correspondientes a un mínimo, medio y máximo valor de los cuatro factores analizados: TA, VA, TF y tf). Enseguida se presentan las ecuaciones cuadráticas como ejemplos, para solo dos de los parámetros estudiados como variables dependientes $\left(R^{2} 0.91\right.$ para humedad y 0.76 para grasa):

$$
\begin{aligned}
& \text { \% Humedad }=4.78-0.63 \mathrm{TA}+0.78 \mathrm{VA}-0.74 \mathrm{TF}-0.87 \mathrm{tf}+1.35 \mathrm{TA}^{2}-0.033 \mathrm{VA}^{2}+0.65 \mathrm{TF}^{2} \\
& +4.561 \times 10^{-3} \mathrm{TABVA}+0.03 \mathrm{TATF}+0.092 \mathrm{TAtf}+0.25 \mathrm{VATF}+0.20 \mathrm{VAtf}-0.12 \mathrm{TFtf} \\
& \text { \%Grasa }=14.42+1.3 \mathrm{TA}-0.96 \mathrm{VA}+1.12 \mathrm{TF}-0.36 \mathrm{tf}+0.25 \mathrm{TA}^{2}-2.90 \mathrm{VA}^{2}-2.17 \mathrm{TF}^{2} \\
& -0.77 \mathrm{TAVA}+1.18 \mathrm{TATF}+1.10 \mathrm{TAtf}-0.67 \mathrm{VATF}+1.03 \mathrm{VAtf}+0.033 \mathrm{TFtf}
\end{aligned}
$$

Donde: TA es la temperatura del aire $\left(35,48\right.$ y $\left.68^{\circ} \mathrm{C}\right)$, VA es la velocidad de aire $(2,4$ y $6 \mathrm{~m} / \mathrm{s})$, TF es la temperatura del aceite $\left(140,160\right.$ y $\left.180^{\circ} \mathrm{C}\right)$, y tf es el tiempo de freído (30, 60 y $\left.90 \mathrm{~s}\right)$.

Empleando los modelos de optimización obtenidos (ecuaciones 2 y 3, entre otras), se determinaron las condiciones de operación que serían necesarias para obtener ciertas características de tres productos comerciales modificados (en grasa sobre todo). Dichas condiciones de operación resultantes, fueron diferentes para cada tostada y se pueden apreciar en la Tabla 5 , donde destacan la velocidad de $2 \mathrm{~m} / \mathrm{s}$ en el secado y $160^{\circ} \mathrm{C}-90 \mathrm{~s}$ en el freído.

Tabla 5: Condiciones de operación (secado y freido), obtenidas a partir de las ecuaciones predictivas.

\section{Parámetros de operación}

\begin{tabular}{lcccc}
\hline Marca & \multicolumn{2}{c}{ Secado } & \multicolumn{2}{c}{ Freido } \\
& $\mathrm{T}\left({ }^{\circ} \mathrm{C}\right)$ & $\mathrm{v}(\mathrm{m} / \mathrm{s})$ & $\mathrm{T}\left({ }^{\circ} \mathrm{C}\right)$ & $\mathrm{t}(\mathrm{s})$ \\
\hline Delicias & 43,62 & 5,99 & 160 & 30,14 \\
Milpa real & 30,63 & 2,00 & 160 & 87,80 \\
Charras & 38,98 & 2,00 & 160 & 90,00
\end{tabular}

Al ser empleadas las condiciones de proceso proporcionadas por la simulación (Tabla 5) en las corridas experimentales de validación (que llevamos a cabo lo mas exactamente posible), se obtuvieron las propiedades de las tostadas (humedad, grasa, color y textura) correspondientes a cada marca, mismas que se incluyen en la Tabla 6; donde se presentan tanto las características teóricas (resultantes de la predicción), como las obtenidas experimentalmente. 
Tabla 6: Resultados predichos y experimentales de cuatro características del producto terminado, para las condiciones de operación establecidas por el programa computacional de optimización.

\begin{tabular}{|c|c|c|c|c|c|c|}
\hline \multirow[t]{2}{*}{ Propiedad } & \multicolumn{2}{|c|}{ Delicias } & \multicolumn{2}{|c|}{ Milpa Real } & \multicolumn{2}{|c|}{ Charras } \\
\hline & Teórico & Práctico & Teórico & Práctico & Teórico & Práctico \\
\hline Humedad (\%) & 6,3 & $6,50 \pm 1.15$ & 3,9 & $4,13 \pm 0,61$ & 2,9 & $2,07 \pm 0,70$ \\
\hline Grasa (\%) & 8,5 & $8,36 \pm 0,48$ & 7,4 & $9,41 \pm 0,23$ & 8,8 & $9,01 \pm 0,51$ \\
\hline$\Delta \mathrm{E}$ & 43,9 & $51.02 \pm 3,77$ & 42,7 & $47,99 \pm 0,82$ & 42,1 & $49,81 \pm 3,30$ \\
\hline Fuerza (N) & 5,8 & $5,80 \pm 0,79$ & 15,8 & $7,21 \pm 0,96$ & 14,3 & $12,54 \pm 3,26$ \\
\hline
\end{tabular}

Mediante un análisis de varianza y realizando la prueba de comparación de Tukey, se determinó que las condiciones de operación obtenidas mediante la optimización, permiten obtener un material alimenticio con características semejantes a las comerciales ( $p>0.05$ ), en lo que respecta a porcentaje de humedad, y textura; sin embargo existe una diferencia significativa en lo que respecta a color $(p<0.05)$, lo cual se puede atribuir a la adición de colorantes que suelen emplearse en los productos comerciales.

Como parte colateral y final de este trabajo, se realizó la evaluación sensorial de 3 tostadas (datos no incluidos), con las siguientes características: una tostada comercial (marca Delicias, TD), y dos tostadas obtenidas en nuestros laboratorio, a partir de diferentes condiciones de proceso. Una tostada producida en condiciones de proceso que simularon a dicha marca (Tabla 6, TS), y una tostada producida en condiciones de proceso $\left(68^{\circ} \mathrm{C}-6 \mathrm{~m} / \mathrm{s}\right.$ en el secado; $140^{\circ} \mathrm{C}-60 \mathrm{~s}$ en el freído, $\mathrm{TL}$ ) para un mínimo contenido de grasa.

Las tostadas de maíz comerciales, resultaron tener mayor aceptación en todos los atributos medidos (color, sabor, textura y aceptación general), seguidas por las que contenían la mínima cantidad de grasa y finalmente las que se elaboraron con los parámetros optimizados. También se observó que las tostadas elaboradas en el laboratorio tuvieron calificaciones muy cercanas en sus atributos sensoriales, lo cual se debe principalmente a que ambos productos se generaron de las mismas tortillas para su elaboración, y que un proceso emuló dicha marca y el otro generó un bajo contenido de grasa. El ANOVA si mostró una diferencia significativa entre los productos analizados sensorialmente.

\section{CONCLUSIONES}

Como resultado de la caracterización de tostadas se observó que en este tipo de productos la humedad es de 1.5 a $6 \%$, la grasa es de 24 a $27 \%$, con parámetros posibles de color entre 23 y 25 para L, 0 a 0.2 para a, de 1.3 a 3.0 para b y una fuerza de fractura entre 5.0 y $8.0 \mathrm{~N}$.

La aplicación del secado, previo al freído, contribuyó a elaborar un producto con un menor contenido de grasa, y con una humedad propia de este tipo de productos. Este fenómeno de deshidratación con aire es el que determinó la cantidad final de aceite en la tostada, una velocidad de aire caliente de $4 \mathrm{~m} / \mathrm{s}$ resultó en un producto con mayor absorción de grasa, aunque la rapidez con la que se evaporó el agua de la tortilla durante el secado fue mayor. Así, la velocidad de evaporación durante la etapa de freído, puede ser controlada mediante el manejo de la velocidad de aire en el secador.

Altas temperaturas de freído, generaron un bajo contenido de humedad final, y la temperatura no tuvo efecto sobre la cantidad de grasa total en el producto. El manejo de $180{ }^{\circ} \mathrm{C}$ como temperatura de freído es la que ocasionó mayor diferencia en el color. La fuerza de ruptura fue mayor al incrementar la temperatura del aceite en la etapa de freído. Los coeficientes de difusión de agua, resultaron similares a otros procesos de freído.

Como resultado de este trabajo, se proporcionan una serie de condiciones de operación que fueron aplicadas experimentalmente, para elaborar un producto de maíz de alto consumo 
(tostadas) que sean potencialmente satisfactorias para el gusto del consumidor, y sobre todo con menor contenido de grasa. La regresión polinomial obtenida puede representar grandes ventajas en la determinación de las condiciones de operación teóricas y en la optimización industrial de este tipo de productos.

Aunque hubo diferencia sensorial entre la tostada comercial y las elaboradas en el laboratorio, las tostadas elaboradas experimentalmente por la combinación de procesos, tuvieron buena aceptación y presentan un menor contenido de grasa en su composición, lo cual se considera una gran ventaja nutricional.

\section{REFERENCIAS}

AACC. Approved Methods of the American Association of Cereal Chemist, AACC, EU. (1986).

AOAC., Official Methods of Analysis, AOAC international, Gaithersburg, MD. EU (2000).

Alto Nivel. Negocios, Finanzas, Economía y Mercadotecnia., Sabritas, Barcel y Pringles crujen en el Mercado, 11 (130), 32-43 (1999).

Balasubramaniam, V.M., Chinnan, M.S., Mallikarjunan, P. y Phillips, R.D., The effect of edible film on oil uptake and moisture retention of a deep-fat fried poultry product. Journal of Food Process Engineering, 20, 17-29 (1997).

Bourne M.C., Food Texture and Viscosity, Concept and Measurement. Academic Press. EU (2002).

Cenkowski, S., Rosete Hidalgo, J., Sosa Morales, M.E. y Vélez-Ruiz, J.F., Superheated steam as pretreatment to reduce oil uptake during deep-fat frying of chicken nuggets. CSBE/SCGAB 2008, Annual Conference. July 13-16. Vancouver, BC, Canada (2008).

Demetriades, K., Guffey, C. y Khalil, M.H., Evaluating the role of honey in fat-free potato chips. Food Technology, Oct, 66-67 (1995).

Esturk, O., Kayacier, A. y Singh, R.K., Reduction of oil uptake in deep fried tortilla chips. Food Science and Technology International, 6 (5), 425-431 (2000).

Gamble, M.H. y Rice, P., Effect of pre-drying on oil uptake and distribution in potato chip manufacture. Journal of Food Science and Technology, 22, 535-540, 542 (1987).

Garayo, J. y Moreira, R.M., Vacuum frying of potato chips. J. Food Engineering, 55, 181-191 (2002).

García-Joffre, J., Argaíz-Jamet, A., Vélez-Ruiz, J.F. y Sosa-Morales, M.E., Doughnuts with reduced fat content based on edible coatings application. Poster: 011-30. IFT Annual Meeting, Julio 28 - 1 Agosto, Chicago, IL, EU (2007).

Hernández, M., Chávez A. y Bourges H., Valor nutritivo de los alimentos mexicanos. Tablas de uso práctico. Instituto Nacional de la Nutrición. México (1974).

INEGI., Índice de volumen físico de producción de la industria de alimentos. www.inegi.com.mx. Adquirido 3 de Marzo de 2009 (2009).

Kawas, M.L. y Moreira, R.G., Characterization of product quality attributes of tortilla chips during the frying process. Journal of Food Engineering, 47, 97-107 (2001).

Larmond, E., Methods for Sensory Evaluation of Food. Publication 1284. Canada (1970).

Lloyd, B.J., Farkas, B.E. y Keener, K.M., Quality comparison of French fry style potatoes produced by oven heating, immersion frying and controlled dynamic radiant heating. Journal of Food Processing and Preservation, 28, 460-472 (2004).

Math, R.G., Velu, V., Nagender, A. y Rao, D. G., Effect of frying conditions on moisture, fat, and density of papad. Journal of Food Engineering, 64, 429-434 (2003).

Mellema, M. Mechanism and reduction of fat uptake in deep-fat fried foods. Trends in Food Science and Technology, 14, 364-373 (2003). 
Mittal, G.S. y Zhang, J., Use of artificial neural network to predict temperature, moisture, and fat in slabshaped foods with edible coatings during deep-fat frying. J. Food Science, 65 (6), 978-983 (2000).

Morales-Pérez, J., Sosa-Morales, M.E. y Vélez-Ruiz, J.F., Study of a combined method (drying and frying) for toasted tortilla manufacturing. 13th World Congress of Food Science and Technology (IUFOST). Nantes, Francia. September 17-21, (2006).

Moreira, R.G., Palau, J. E., y Sun, X. 1995a. Deep-fat frying of tortilla chips: An Engineering Approach. Food Technology, 4, 146-150 (1995a).

Moreira, R.G., Palau, J. y Sun, X., Simultaneous heat and mass transfer during the deep fat frying of tortilla chips. Journal of Food Process Engineering, 18, 307-320 (1995b).

Moreira, R.G., J. Palau, V.E. Sweat, y Sun, X., Thermal and physical properties of tortilla chips as a function of frying time. Journal of Food Processing and Preservation, 19, 175-189 (1995c).

Moreira, R.G., Sun, X. y Chen, Y., Factors affecting oil uptake in tortilla chips in deep-fat frying. Journal of Food Engineering, 31: 485-498 (1997)

Moreira, R.G., Castell-Perez, M.E. y Barrufet, M.A., Low-fat tortilla chips. En Deep-fat drying. Fundamentals and applications. Pp. 275-313. Aspen Publishers Inc. Gaithersburg, ML, EU (1999).

Moyano, P.C. y Pedreschi, F., Kinetics of oil uptake during frying of potato slices: Effect of pre-treatments. Food Science and Technology/ LWT, 39, 285-291 (2006).

Moyano, P.C., Ríoseco, V. y González, P.A. Kinetics of crust color changes during deep-fat frying of impregnated French fries. Journal of Food Engineering, 54, 249-255 (2002).

Okos M.R., Narsimhan G., Singh R.K. and Weitnauer A.C., Food dehydration. In Handbook of Food Engineering (D.R Heldman. and D.B. Lund; eds.). Pp. 437- 562. Marcel Dekker, Inc. NY, NY, EU (1992).

Pinthus, E. J., Weinberg, P. y Saguy, I.S., Gel strength in restructured potato products affects oil uptake during deep-fat frying. Journal of Food Science, 57 (6), 1359-1360 (1992).

Rahman, M.S. y Perera, C.O., Drying and food preservation. En Handbook of Food Preservation (M.S. Rahman. Ed.). Pp. 423. CRC Press. Boca raton, FL, EU (2007).

Rubnov, M. y Saguy, I.S., Fractal analysis and crust water diffusivity of a restructured potato product during deep fat frying. Journal of Food Science, 62 (1), 135-137 (1997).

Sinram, R.G. y Hartman, G.J., Flavored vegetable oils as a substitute for frying tallow in deep-frying applications. Food Technology, Junio, 90-94 (1989).

Sosa-Morales, M.E. y Vélez-Ruiz, J.F., Effects of frying and combined treatments on fried foods. En Food Processing and Engineering Topics. (M.E. Sosa-Morales y J.F. Vélez-Ruiz, eds.). Pp. 437- 562. Ed. Nova Pub. Nueva York, EU (2009).

Sosa-Morales, M.E., Orzuna-Espíritu, R. y Vélez-Ruiz, J.F., Mass, thermal and quality aspects of deep fat frying of pork meat. Journal of Food Engineering. 77, 731-738 (2005)

Treybal, R.E., Mass-Transfer Operations. Pp. 665. McGraw-Hill Book Co. NY, EU (1980). 
